sicht auf ein gutes funktionelles Ergebnis und einen Trend $\mathrm{zu}$ erniedrigter Mortalität und nicht erhöhter symptomatischer intrazerebraler Blutungen (SICH), so Röther. Zu einem ähnlichen Ergebnis kamen Badhiwala et al. [8], diesmal aus neurologischer Sicht.

„Die Ergebnisse der fünf neuen Studien zur kombinierten IVT und MT für sich betrachtet sind mit einer ,number needed to treat' von 5 für die modifizierte Rankin-Skala (mRS) nach 90 Tagen spektakulär“, kommentierte Röther. Die neuen Herausforderungen sieht er darin, geeignete Patienten rasch und gezielt mittels Nativ-CT und CT-Angiografie zu selektieren. Zudem müsse die Infrastruktur optimiert werden, damit alle Patienten möglichst schnell in entsprechend qualifizierten Schlaganfallzentren behandelt werden können. Ute Ayazpoor

Schlaganfall akut (Joachim Röther), 8. Neurologie-Update-Seminar, Mainz, 19.-20.2.2016
Literatur

1. Berkhemer OA et al. N Engl J Med 2015; 372: $11-20$

2. Campbell BC et al. M Engl J Med 2015;372: 1009-18

3. Jovin TG et al. N Engl J med 2015; 372: 2296-306

4. Goyal M et al. N Engl J Med 2015; 372: 1019-30

5. Saver JL et al. N Engl J Med 2015; 372: 2285-95

6. Prabhakaran S et al. JAMA 2015; 313: 1451-62

7. Sardar P et al. Eur Heart J 2015; 36: 2373-80

8. Badhiwala JH et al. JAMA 2015; 314: 1832-43

\title{
Positive Drucktherapie bringt nichts bei Morbus Menière
}

In der Hals-Nasen-Ohren-Heilkunde wird der Meniett-Niedrigpulsgenerator oft mit dem Ziel eingesetzt, den endolymphatischen Hydrops zu reduzieren. Dass diese Methode den Schwindel nicht bessert und sogar kontraproduktive Effekte haben kann, zeigen die Ergebnisse einer aktuellen Cochrane-Metaanalyse.

$\mathrm{M}$ it dem Meniett-Druckpulsgenerator können computerkontrolliert Sequenzen mit niedrigen Druckimpulsen (Mikrodruck) verabreicht werden, die - so die Vermutung - zum vestibulären System weitergeleitet werden, um dann den endolymphatischen Hydrops zu reduzieren, erläuterte Professor Marianne Dieterich, Direktorin der Klinik und Poliklinik für Neurologie der Ludwig-Maximilians Universität München. Eine Cochrane-Metaanalyse ging der Wirksamkeit dieses in der Hals-NasenOhren-Heilkunde gerne eingesetzten Verfahrens nun auf den Grund [Sonsbeck van $S$ et al. Cochrane Library 2015; 3: 1 - 49]. Analysiert wurden fünf rando- misierte klinische Studien mit insgesamt 265 Menière-Patienten, die die positive Drucktherapie mithilfe eines MeniettVerfahrens einer ähnlichen Methode mit Placebo gegenüberstellten.

Die meisten Studien ergaben keinen signifikanten Unterschied in der Schwindelkontrolle (primärer Endpunkt, gemessen anhand von Schwindelskalen oder der Anzahl der Tage mit Schwindel) zwischen der Meniett-Therapie und Placebo. Die sekundären Outcomes konnten zum Teil nicht analysiert werden, sei es weil sie nicht erhoben wurden oder die Daten inadäquat waren - wie etwa bei der Schwere von Tinnitus oder Völlegefühl im Ohr. Bei der Hörminderung, die in die Analyse einging, war die positive Drucktherapie sogar unterlegen. Patienten in der Placebogruppe hörten um 7,38 $\mathrm{dB}$ besser als die Patienten in der Meniett-Gruppe, berichtete Dieterich.

Angesichts dieser Ergebnisse riet die Neurologin: „Bitte das Device nicht einsetzen, auch wenn HNO-Kollegen es gerne tun. Die Wirksamkeit auf den Morbus Meniere oder das Meniere-Syndrom ist nicht nachgewiesen." Die Therapie sei zwar minimalinvasiv, aber auch das Einsetzen einer TympanotomieRöhre sei mit Risiken der Anästhesie, dem generellen Risiko einer Operation, dem spezifischen Risiko einer Otorrhoe und Tympanosklerose verbunden.

Ute Ayazpoor

Neuroophtalmologie/Schwindel (Marianne Dieterich), 8. Neurologie-Update-Seminar, Mainz, 19. -20.2 .2016$

\section{Chronischer Schmerz ist jetzt für ICD11 definiert}

Eine lange überfällige gute Nachricht für die Forschung - und die Versorgung von chronischen Schmerzpatienten: In der Neuauflage der International Disease Classification (ICD-11) konnte man sich erstmals auf eine international gültige Klassifikation von chronischen Schmerzen einigen. Dass das Expertengremium der Internationalen Schmerzgesellschaft IASP, anderen internationalen Fachgesellschaften und der WHO so lange um eine einheitliche Taxonomie von chronischen
Schmerzen gerungen hat, liegt an der ätiologischen und phänotypischen Vielfalt des Schmerzes, die die Zusammenarbeit vieler unterschiedlicher klinischer Fächer erzwinge, so Professor Arne May, Hamburg. In der ICD11 [Treede R-D et al. Pain 2015; 156: 1003-7] sind nun sechs verschiedene chronische Schmerzformen definiert: primärer chronischer Schmerz, chronischer Krebsschmerz, chronischpostchirurgischer Schmerz, chronischneuropathischer Schmerz, chronischer
Kopfschmerz, chronischer viszeraler Schmerz und chronischer muskuloskelettaler Schmerz. Die strukturierte und gebündelte Klassifikation macht es endlich möglich, dass Forschungsvorhaben homogenisiert - und damit die Voraussetzungen für eine weitere Verfeinerung der Klassifikation geschaffen werden, kommentierte May. Ute Ayazpoor

Schmerz/Kopfschmerz (Arne May), 8. Neurologie-Update-Seminar, Mainz, 19.-20.2.2016 